Case Report

\title{
Irreversible visual loss and posterior uveitis as the initial manifestation of Behcet's Disease
}

\author{
Annamalai R. ${ }^{1}$, John A.A. ${ }^{2}$, Muthayya M. ${ }^{3}$ \\ ${ }^{1}$ Prof. Dr. Radha Annamalai, Professor of Ophthalmology, ${ }^{2}$ Dr. Ansu Ann John, Postgraduate (MS Ophthalmology), ${ }^{3}$ Prof. M. \\ Muthayya, Professor of Ophthalmology, Department of Ophthalmology, Sri Ramachandra Institute of Higher Education and \\ Research, Porur, Chennai, India.
}

Corresponding Author: Prof. Dr. Radha Annamalai, Professor of Ophthalmology, Department of Ophthalmology, Sri Ramachandra Institute of Higher Education and Research, Porur, Chennai, India. Mail: drradhasrmc@gmail.com

\begin{abstract}
We report a rare case of rapid onset, severe visual loss in a young male patient with Behcet's disease who had ocular disease as an initial manifestation. Vision was perception of light with inaccurate projection in both eyes. Anterior segment showed no inflammation. Fundus examination revealed vitritis, total optic atrophy, branch retinal vein occlusion and cellophane maculopathy. Visual evoked potential showed delayed P 100 responses in the left eye suggestive of left anterior visual pathway conduction defect. Systemic examination showed no active symptom complex. There was no response to oral corticosteroids or immunosuppressive drugs. Though rare in the Indian population, Behcet's disease can be severe, progress rapidly and is less responsive to conventional treatment as in our patient. This case is presented for its rarity, as ocular features were the initial and only manifestation. Despite immediate therapy with corticosteroids and immunosuppressives, continued inflammation resulted in visual loss.
\end{abstract}

Keywords: Behcet's, Immunosuppressives, Corticosteroids, Retinitis, Optic atrophy

\section{Introduction}

Behcet's disease is a rare, chronic, relapsing disease characterized by mucocutaneous ulcerations, genital ulcers and eye inflammation [1]. The genetic marker associated with this disorder is HLA- B51 [2]. Major systemic symptoms include oral aphthous ulcers, genital ulcers, ocular features and skin manifestations [3]. Ocular features may appear in the form of anterior uveitis, posterior uveitis and retinal vasculitis in about $70 \%$ of patients [4].

It may lead to visual loss with frequent recurrences. Men are affected mostly, with almost a quarter of these patients suffering from severe visual loss. Even though the eye symptoms might be the drastic complication of this disease, the initial presenting signs may be oral ulcers in about threefourths of these patients. Changes that occur in the buccal mucosa are painful erythematous oral ulcers. Multi organ system involvement is associated with kidney, gastrointestinal disorders, respiratory and central nervous system changes. Genital ulcers are less frequent and have a propensity for scar formation. Late manifestations include central nervous system involvement, mostly in men in about $5-10 \%$ cases [5].

Manuscript received: $20^{\text {th }}$ November 2019

Reviewed: $30^{\text {th }}$ November 2019

Author Corrected: $6^{\mathrm{h}}$ December 2019

Accepted for Publication: 11 ${ }^{\text {th }}$ December 2019
Deep vein thrombosis is a common finding. Multiple joint involvement including ankles, wrists, knees, hips and elbows are seen in roughly half of these patients. The gastrointestinal tract is involved less commonly in the Mediterranean population and more commonly in Japan. In 1937, a Turkish dermatologist noticed a repeating pattern of constellation of signs, which he investigated further and described later in his book the Archives of Dermatology and Venereal disease [6].

Most often noted in Turkey, among the other Mediterranean countries, its prevalence is lesser in the Western population and uncommon in India. Treatment is aggressive with continuous use of immunosuppressives where permitted, along with corticosteroids. This case is presented for its rarity due to the initial and only ocular manifestations, no systemic featuresand the non-responsive nature in spite of aggressive management using immunosuppressives and corticosteroids, leading to severe visual morbidity. Both the clinical and pathological diagnosis is difficult as there are no specific guidelines. For the classification of Behcet's disease a new diagnostic criterion to guide the diagnosis has been improvised in which ocular signs are incorporated as an essential criterion unlike the previous classification where eye signs need not always exist for a diagnosis [7]. 
Case Report

\section{Case Report}

A34 year old healthy, male patient presented with complaints of diminution of vision and occasional episodes of redness in both eyes since 4 years. He had been diagnosed with conjunctivitis and was treated with antibacterial eye drops by the local ophthalmologist. He suffered from no other comorbidities at that time. His symptoms worsened and at the time of presentation to us, he had perception of light but inaccurate projection in the left eye, $<6 / 60$ in the right eye with 30 -degree exophoria in the right eye. On slit lamp examination, the anterior segment showed no cells or flare and the cornea was clear with no old or fresh keratic precipitates. Pupil was round and sluggishly reacting in both eyes with no relative afferent pupillary defect (RAPD). Fundus examination with indirect ophthalmoscopy revealed grade 1 vitritis, total optic atrophy, branch retinal vein occlusion and epiretinal membrane in both eyes (Figure 1). Visual evoked potential (VEP) showed delayed P 100 responses in the left eye suggestive of a left anterior visual pathway conduction defect. Intraocular pressure was normal in both eyes. OCT showed an irregular outer plexiform layer with areas of elevation and a generalized thinned out retina.

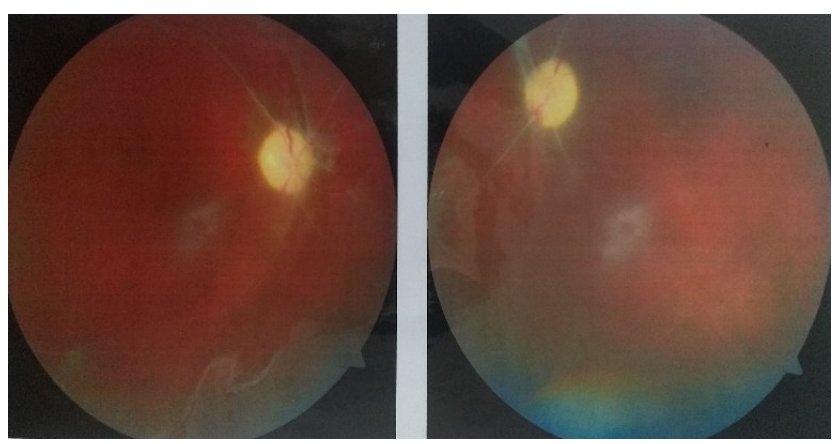

Fig-1: Fundus examination showing vitritis, total optic atrophy, branch retinal vein occlusion and epiretinal membrane in both eyes.

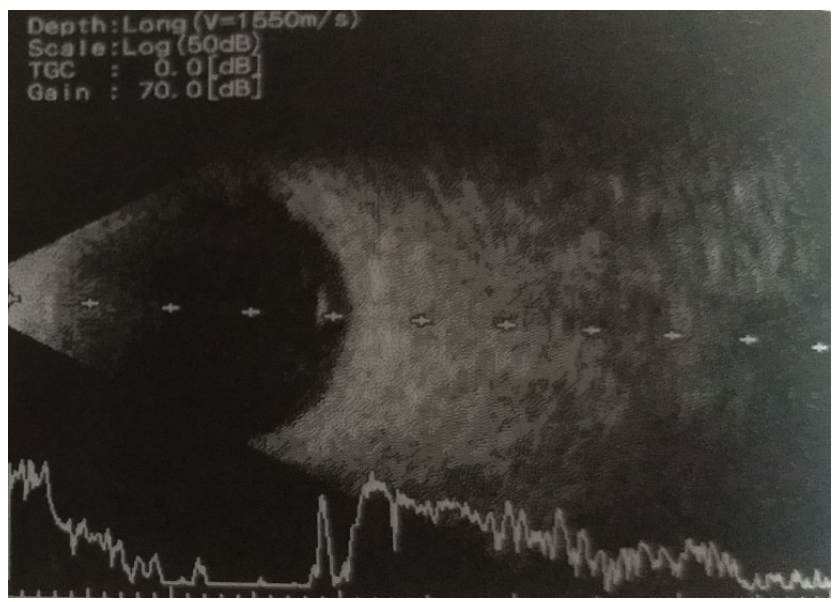

Fig-2: B scan showing posterior vitreous detachment.

B scan showed posterior vitreous detachment and a decreased choroidal thickness probably suggesting a chronic choroidal inflammation with atrophy (Figure 2). HLA B5 was positive. Renal, radiological and cardiac evaluation was normal. The present study continued systemic investigations to rule out viral disease as a cause of posterior uveitis in this patient. This case is being reported for several reasons. Ocular Behcet's is commonly known to present with systemic involvement prior to ocular features but our patient had irreversible, total visual loss following severe retinitis and vasculitis despite treatment. Besides, the uveitis in this patient had no anterior segment involvement and had occurred with an atypical initial presentation of conjunctival or episcleral congestion.

The patient did not satisfy the diagnostic criteria for Behcet's disease and a differential diagnosis of viral retinitis was made. However, microbiological testing for viral disease, which had been done, was negative. Laboratory markers for posterior uveitis such as tuberculosis, sarcoidosis and syphilis were not contributory. Rheumatological workup and blood investigations did not reveal positive antibodies. 


\section{Case Report}

\section{Discussion}

Specific tests to diagnose Behcet's disease are not yet established and guidelines do not always help in the diagnosis especially in atypical presentation. A likely diagnosis can be made based on laboratory tests, imaging, multispecialty consultation and clinical examination. The development of all features may take many months or even years to develop and Behcet's disease needs to be considered in any patient with floaters, pain or visual loss and posterior uveitis [8].

The disease is recognized to have multi organ involvement affecting the intestine, joints, blood vessels, nerves and mucocutaneous junctions. Frequency of Behcet's uveitis ranges from 50 to $85 \%$ and the eyes usually get involved about 2-3 years after systemic features [9]. The onset of systemic disease almost always occurs prior to ocular disease. However the presence of initial eye inflammation has been reported in only $10-15 \%$ of patients [10]. The presence of bilateral involvement ranges from $78 \%$ to $95 \%$ and the mean age of presentation ranged from 11 to 37 years [11]. The revised international criteria for $\mathrm{BD}$ given in 2010, includes recurrent oral ulcers at least 3 times per year, recurrent genital ulcers, skin lesions such as erythema nodosum or papulopustular rash, ocular inflammation, thromboembolism, central nervous system involvement and a positive pathergy test.

The prevalence of HLA B5 is now found to be high in some subsets of the Indian population which is comparable to Mediterranean and the Middle east where the disease is prevalent [12]. Uveitis is not common in Behcet's disease but relapsing remitting panuveitis and retinal vasculitis are the most frequent manifestation. Anterior uveitis is always non granulomatous and acute but is associated with a hypopyon in one third of patients. The presence of a hypopyon usually indicates an associated posterior segment inflammation. Vitritis is a consistent feature and severe vitreous haze occurs in severe posterior segment inflammation. Disc swelling, retinal infiltrates, branh retinal vein occlusions and exudative retinal detachments are other ophthalmic manifestations [13]. An important diagnostic sign is superficial, transient retinal infiltration and spontaneous resolution of uveitis.

They disappear without scarring but may recur. Periphlebitis which may be occlusive or leaky in nature may be associated with arteriolitis and capillaritis. Sheathing due to gliosis indicating previous vasculitis can be visualized as occurred in our patient. Recurrent venous occlusions can involve central retinal vein or smaller venules. Fundus fluorescein angiography demonstrates retinal capillary non perfusion. Neovascularisation can develop secondary to retinal ischemia and has been reported in $13 \%$ of patients
[14].Uncontrolled or severe uveitis can cause complications such as posterior synechiae, secondary glaucoma, cataract, macular oedema, retinal tears, retinal detachment, optic atrophy and phthisis bulbi.In the late stages after resolution of inflammation, vitreous is totally clear.

Vitreous haemorrhage, macular oedema, necrotising retinitis, retinal periaretiritis and periphlebitis can cause visual loss in these patients. The clinical presentation and features of Behcet's disease seem to vary based on geographic and genetic influences. In India, less of ocular and more of joint involvement has been reported. To our knowledge, isolated ocular involvement has not been reported in the Indian population. This patient has been followed up for the past 7 years but did not show evidence of systemic disease. The association of ocular involvement in some of these patients has now been found to depict a more severe form of the disease.

In the present study, the patient did not have any iridocyclitis and the typical features such as hypopyon and non-granulomatous anterior uveitis did not exist. Vitreous floaters and haze suggested inflammation and the presence of branch retinal vein occlusion indicated a severe vasculitis. Non-perfusion of peripheral retina and neovascularisation can lead to vitreous haemorrhage and result in vision loss [15]. It was found that the bilateral optic atrophy, and this could be due to generalized loss of functional retinal layers. The visually evoked potential test showed a deficient anterior visual pathway, which could occur secondary to spread of inflammation and neurological involvement. The absence of relative afferent papillary defect and presence of optic atrophy in our patient is a rare sign and could actually mislead the treating ophthalmologist about the state of the optic disc. Even after intensive treatment, blindness can occur in upto $50 \%$ of patients due to fundus involvement [16]. Behcet's disease responds well to immunosuppressive treatment and steroids in more than half of these cases [17].

Immunomodulatory therapy for the treatment of posterior uveitis is indicated in all patients with Behcet's disease. Combination therapy with corticosteroids and azathioprine or cyclosporine is used as first line treatment. In resistant or non-responsive patients infliximab or interferon alfa is used and is of benefit in refractory ocular Behcet's, when it was not controlled by other regimens. Another biological agent etanercept has been found useful in these cases, though not as much as infliximab [18].

For refractory cases other alternative drugs are adalimumab, rituximab and TNF alpha regulator have been reported to have variable success [19]. 
Previous evidence proves that IL-17, IL-21, and TGF- $\beta$ markers correlated with disease activity. Thus, the aim to target these cytokines may constitute a promising novel therapy [20]. IL-21 blocking agents have been used in severe or refractory $\mathrm{BD}$, as it is known that it restores the altered balance between Th17 cells and regulatory $\mathrm{T}$ cells (Tregs) [21]. Childhood Behcet's [22] has been reported to present with uveitis and retinitis. Currently, cyclosporine is regarded superior in the treatment of refractory or severe eye disease, the major contraindication being neurotoxicity development [23]. Visual prognosis depends on the severity and frequency of the uveitic attacks.

Although a single episode may result in permanent loss of vision, recurrent attacks as a cause for poor outcome is more common. It has been reported that young male patients have more acute inflammation, severe complications and worse visual outcome. Recent reports suggest that an improvement in the environment in a stable genetic makeup causes milder disease in certain populations (Japan) [24]. Visual prognosis is much better in those with early aggressive treatment and compliance during follow up to monitor for complications.

Uveitis in $\mathrm{BD}$ is an absolute indication for systemic immunomodulation. Isolated anterior uveitis requires local cycloplegics and corticosteroids. Vitreous cells and leakage from retinal vessels detected on fluorescein angiography requires immediate treatment with immunomodulatory drugs even in the absence of obvious posterior segment features. Systemic corticosteroids need to be used only in active vision threatening uveitis. Tapering has to be done carefully as rebound attacks can be very severe.

Monotherapy with azathioprine and cyclosporine is not as effective. A recent recommendation based on evidence in treatment of Behcet's disease states that corticosteroids and azathioprine can be used in mild inflammation but in severe uveitis, cyclosporine or infliximab is required. Interferon alfa in a single infusion of $5 \mathrm{mg} / \mathrm{kg}$ rapidly reduces inflammation and has long term remission in $90 \%$ of patients [25]. It is useful in sever attacks when there is a risk of structural damaga and permanent vision loss.

However, infusions may be required at short intervals and cost of treatment with infliximab is a limiting factor. The present used corticosteroids and azathioprine with corticosteroids in all our patients with Behcet's uveitis. The current study used infliximab onlyin patients who had not responded to the triple agent regimen.

Uveitis in BD is a therapeutic challenge. Treatment always needs to be done in concurrence with a physician. In some situations, such as in our patient where only ocular features were present, it can be a diagnostic dilemma. The rapid spread of active retinitis, vitritis and vasculitis are the pointers to a diagnosis of $\mathrm{BD}$. Behcet's disease remains an immunologic mystery, a clinical and a therapeutic challenge to internists, immunologists, and ophthalmologists alike.

The importance of long-term follow-up remains crucial in the prognosis of the disease. With further collaborative research and understanding of the disease and its patterns of organ affection and/or avoidance, the present study may reach new classifications which will help us better plan our treatment and follow-up regimens and even develop prophylactic options.

The present case report recommends that predictors for severity of ocular diseases mentioned in this study be implemented in the initial assessment for disease and its diagnostic criteria and eventually be taken into consideration when designing the treatment plan.

\section{Conclusion}

Ocular involvement in Behcet's disease is an emergency and aggressive treatment is required in all patients to prevent visual morbidity. The presence or onset of visual loss in these patients points towards retinal or optic nerve involvement and carries a poor prognosis if immediate therapy is not started. Systemic features may or may not be present and a multispecialty approach will be of benefit in the management of these patients.

Funding: No funding sources

Conflict of interest: None declared

Ethical Approval: Not required

\section{References}

1. Scherrer MAR, Rocha VB, Garcia LC. Behçet's disease: review with emphasis on dermatological aspects. Anais Brasileiros de Dermatologia. 2017;92(4):452-464. doi: 10. 1590/abd1806-4841.20177359.

2. McCluskey P, Powell PRJ. The eye in systemic inflammatory diseases. The Lancet. 2004; 364(9451):21252133. doi: 10.1016/S0140-6736(04)17554-5.

3. Alpsoy E, Donmez L, Onder M, Gunasti S, Usta A, Karincaoglu Y, et al., Clinical features and natural course of Behçet's disease in 661 cases: a multicentre study. Brit J Dermatol. 2007;157(5):901-906. doi: 10.1111/j.1365-2133. 2007.08116.x.

4. Prete M, Guerriero S, Dammacco R, Fatone MC, Vacca A, Dammacco F, et al. Autoimmune uveitis: a retrospective analysis of 104 patients from a tertiary reference center. J Ophthalmic Inflamm Infect. 2014;24;4(1):17. doi: 10.1186 /s 12348-014-0017-9. 
5. Hazirolan D, Sungur G, Duman S., Demographic, clinical, and ocular features in patients with late-onset Behçet disease. Ocular Immunol Inflam. 2012;20(2):119124. doi: 10.3109/09273948.2012.655882.

6. UStün C. A famous Turkish dermatologist, Dr. Hulusi Behçet. Eur J Dertmatol. 2002;12(5):469-470.

7. Davatchi F, Sadeghi Abdollahi B, Chams-Davatchi C, Shahram F, Shams H, Nadji A, et al. The saga of diagnostic /classification criteria in Behcet's disease. Int J Rheumat Dise. 2015; 18(6): 594-605. doi: 10.1111/1756-185X. 12520 .

8. Tugal-Tutkun I, Onal S, Altan-Yaycioglu R, Altunbas $\mathrm{HH}$, Urgancioglu M., Uveitis in Behçet disease: an analysis of 880 patients. Am J Ophthalmol. 2004;138(3):373-380. doi: 10.1016/j.ajo.2004.03.022.

9. Nussenblatt RB. Uveitis in Behçet's disease. Int Rev Immunol. 1997;14(1):1467-1479. doi: 10.3109/0883018 9709116845.

10. Rohatgi J, Singal A. Ocular manifestations of behcet's disease in Indian patients. Ind J Opthalmol. 2003;51(4): 309-313.

11. Nobuyoshi K, Akiko M, Miles RS., Hormoz C, Daiju I, Shigeaki O. Ocular features of Behçet's disease: An international collaborative study. Br J Ophthalmol. 2007; 91 (12): 1579-1582. doi: 10.1136/bjo.2007.123554.

12. Michelson JB, Chisari FV. Behçet's disease. Surv Ophthalmol. 1982;26(4):190-203. doi: https://doi.org/ 10. 1016/ 0039-6257(82)90079-0.

13. Biswas J, Kharel Sitaula R, Multani P.Changing uveitis patterns in South India. Comparison between two decades. Indian J Ophthalmol. 2018; 66(4):524-527. doi: 10.4103/ ijo. IJO 85117.

14. Sachdev N, Kapali N, Singh R, Gupta V, Gupta A. Spectrum of Behçet's disease in the Indian population. Int Ophthalmol. 2009;29(6):495-501. doi: 10.1007/s10792008-9273-8

15. Okada AA. Behçet's disease: general concepts and recent advances. Curr Opin Ophthalmol. 2006; 17(6):551556. doi: 10.1097/01.icu.0000247411.59213.bb.
16. Atmaca LS. Fundus changes associated with Behçet's disease. Graefes Arch Clin Exp Ophthalmol 1989; 227(7): 340-344. doi: 10.1007/ bf 02169409.

17. Evereklioglu C. Current concepts in the etiology and treatment of Behçet disease. Sur Ophthal-mol. 2005; 50 (4): 297-350. doi: 10.1016/j.survophthal. 2005.04. 009

18. Handa $\mathrm{T}$, Tsunekawa $\mathrm{H}$, Yoneda $\mathrm{M}$, Watanabe $\mathrm{D}$, Mukai T, Yamamura M. Long-term remission of ocular and extraocular manifestations in Behcet's disease using infliximab. Clin Exp Rheumatol. 2011; 29(4 Supp 67): S58-S63.

19. Khera TK, Dick AD, Nicholson LB. Mechanisms of TNF $\alpha$ regulation in uveitis: focus on RNA-binding proteins. Prog Ret Eye Res. 2010;29(6):610-621. doi: 10. 1016/ j. preteyeres.2010.08.003.

20. Comarmond C, Wechsler B, Bodaghi B, Cacoub P, Saadoun D. Biotherapies in Behçet's disease. Autoimmun Rev. 2014; 13(7):762-769. doi: 10.1016/j.autrev. 2014. 01.056

21. Atzeni F, Sarzi-Puttini P, Doria A, Boiardi L, Pipitone N, Salvarani C. Behçet's disease and cardiovascular involvement. Lupus. 2005;14(9):723-726. doi: 10.1191/ 0961203305lu2208oa.

22. Ozdal PC, Ortac S, Taskintuna I, Firat E. Long term therapy with low dose cyclosporin a in ocular Behcet's disease. Doc Ophthalmol. 2002;105(3): 301-312. doi: 10. 1023/ a:1021227019915.

23. Tugal-Tutkun I, Urgancioglu M. Childhood-onset uveitis in Behcet disease: a descriptive study of 36 cases. Am J Ophthalmol. 2003;136(6):1114-1149. doi: 10.1016/ S0002-9394 (03)00791-8.

24. Rathinam SR, Namperumalsamy P. Global variation and pattern changes in epidemiology of uveitis. Indian $\mathrm{J}$ Ophthalmol. 2007;55(3):173-83. doi: 10.4103/0301-4738. 31936.

25. McNally TW, Damato EM, Murray PI, Denniston AK, Barry RJ. An update on the use of biologic therapies in the management of uveitis in Behcet's disease: a comprehensive review. Orphanet J Rare Dis. 2017; 12(1): 130. doi: 10.1186/s13023-017-0681-6.

\section{How to cite this article?}

Annamalai R, John A.A, Muthayya M. Irreversible visual loss and posterior uveitis as the initial manifestation of Behcet's Disease. Trop J Ophthalmol Otolaryngol.2019;4(8):502-506.doi:10.17511/jooo.2019.i08.08 